\title{
A Multi-Systems Approach to Human Movement after ACL Reconstruction: The Integumentary System
}

\author{
Kathryn Lucas, PT, DPT, PhD, SCS, OCS ${ }^{1}$, Patricia Todd, MD², Brandon M Ness, PT, DPT, PhD, SCS ${ }^{3}$ \\ ${ }^{1}$ Kosair Charities Center for Pediatric NeuroRecovery, University of Louisville; Kentucky Spinal Cord Injury Research Center; Department of \\ Neurological Surgery, University of Louisville, ${ }^{2}$ Department of Pediatrics, University of Louisville School of Medicine, Norton Children's Pediatric \\ Dermatology, ${ }^{3}$ Doctor of Physical Therapy Program, Tufts University School of Medicine \\ Keywords: anterior cruciate ligament, anterior cruciate ligament reconstruction, integumentary system, movement system, rehabilitation \\ https://doi.org/10.26603/001c.29454
}

\section{International Journal of Sports Physical Therapy}

Vol. 17, Issue 1, 2022

\begin{abstract}
Postoperative management of anterior cruciate ligament (ACL) reconstruction has traditionally focused on the evaluation and intervention of musculoskeletal components such as range of motion and patients' reports of function. The integumentary system can provide early indications that rehabilitation may be prolonged due to protracted or poor healing of the incision sites. Full evaluation of the reconstruction over time, including direction of the incisions, appearance of surgical sites, level of residual innervation, and health of the individual should be considered when determining time-based goals and plans for returning an athlete to activity. Skin care techniques should be used to minimize strain and promote wound healing at the surgical sites, which in turn allows for implementation of other interventions that target other body systems such as locomotion, strength training, and cardiopulmonary conditioning. The integration of the integumentary system with cardiovascular, neurological, and muscular systems is required for a successful return to activity. A multi-physiologic systems approach may provide a unique viewpoint when aiming to attain a greater appreciation of the integumentary system and its integration with other body systems following ACL reconstruction. The purpose of this clinical commentary is to discuss integumentary considerations within a multi-physiologic systems approach to human movement after ACL reconstruction, including an anatomical review, key elements of assessment, and integrated intervention strategies.
\end{abstract}

\section{Level of Evidence}

5

\section{INTRODUCTION}

In 2013, the American Physical Therapy Association (APTA) adopted a new vision statement that called for physical therapists to 'transform society by optimizing movement to improve the human experience.' ${ }^{1}$ A product of this vision was the development of the movement system, where the APTA described the movement system as a term to represent the interaction of a collection of systems that ultimately contribute to human movement. ${ }^{2}$ Several physical therapy publications (position papers, editorials, commentaries) have voiced support for using the movement system as a foundation for physical therapist practice, education, and research. ${ }^{3-6}$ One of the most critical elements of the proposed movement system model is the integration of physiological systems and the multi-system contributions to purposeful, efficient human movement.

The integumentary system (IS) is rarely the focus of rehabilitation after anterior cruciate ligament reconstruction (ACLR); however, impairments of the IS can contribute to deficits in other physiologic systems that lead to movement limitations. The IS contributes to thermoregulation during cardiovascular training, ${ }^{7}$ sensation for neuromuscular control, ${ }^{8}$ and fascial mobility for range of motion of the

\footnotetext{
a Correspondence:

Brandon Ness, PT, DPT, PhD

Doctor of Physical Therapy Program

Tufts University School of Medicine

136 Harrison Ave

Boston, MA, 02111

Email: Brandon.Ness@tufts.edu
} 

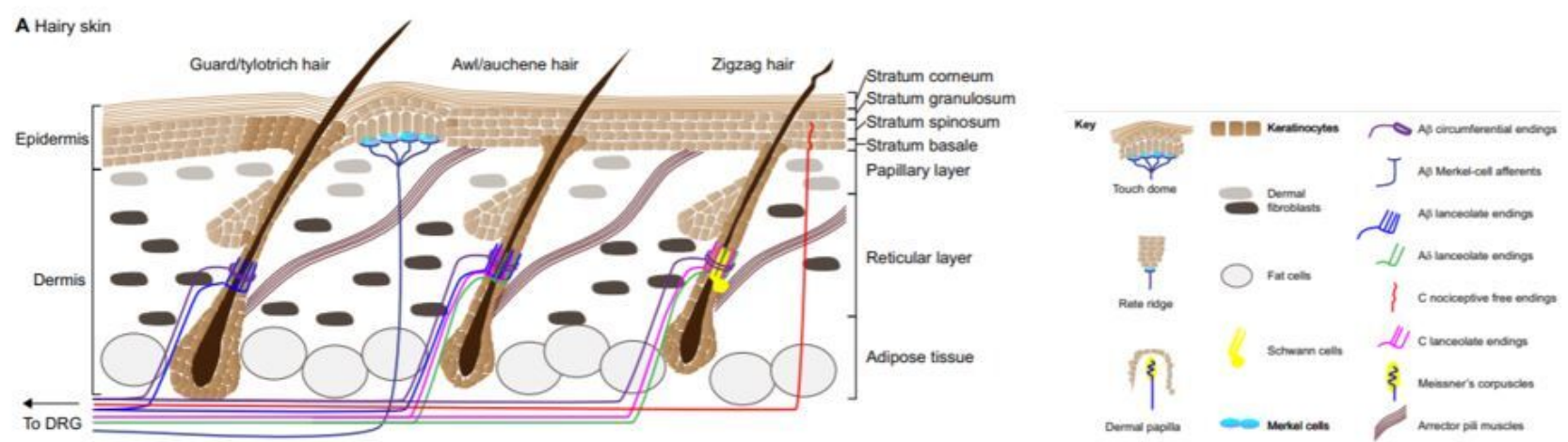

Figure 1. Anatomy of the Skin

Used with permission from Development. Jenkins BA, Lumpkin EA. Developing a sense of touch. Development. 2017;144(22):4078-4090. doi:10.1242/dev.120402

joint. ${ }^{9,10}$ Despite traditional rehabilitation protocols that focus on incision site healing for approximately two weeks, ${ }^{10,11}$ the IS continues to contribute to an athlete's rehabilitation for months after surgery. These contributions illustrate the crucial role of the IS in supporting optimal movement strategies in the long term. The purpose of this clinical commentary is to discuss integumentary considerations within a multi-physiologic systems approach to human movement after ACLR, including an anatomical review, key elements of assessment, and integrated intervention strategies. Additionally, this commentary will identify potential warning signs indicative of impaired IS healing after ACLR.

\section{REVIEW OF ANATOMY}

The IS is one of the largest organs of the body. It functions as a structural barrier as well as providing thermoregulation, sensation, and balancing of hydration and electrolytes. ${ }^{12}$ Age, fitness, nutrition, and genetics all play a large role in the health of the skin. ${ }^{13}$ The epidermis is the outermost layer, followed by the dermis, the most critical layer in wound healing. The dermis gives the skin its strength and elasticity while containing superficial vessels and nerves. Beneath the dermis lies the third portion of the IS: adipose tissue. Within the subcutaneous fat, there are larger nerves and vascular structures. ${ }^{12}$ (Figure 1)

Skin thickness, innervation, adnexal structures, and elasticity vary depending on the location in the body. ${ }^{13} \mathrm{Ar}$ eas of the knee with prominent and superficial bony structures, such as the patella, tibial tuberosity, and anterior crest of the tibia have a mobile dermal layer that is relatively inelastic with minimal fat composition. ${ }^{13}$ Subcutaneous bursae reduce friction between the dermis and fascia while Havers' glands ("fat pads") surround the patellar tendon.

\section{SURGICAL RECONSTRUCTION OF THE ACL}

There are currently four common surgical reconstruction techniques used for the ACL. The bone-patellar-tendonbone (BPTB), hamstring (HS), quadriceps tendon (QT), and allograft are the most frequently used ACLR grafts. ${ }^{9,14}$ The organization of the IS and the impact scarring has on the body varies depending on the incision site's location, the depth and direction of the incision, the underlying tissues (muscle, fascia, tendon, bone, etc.), and the overall health (including blood flow and mobility) of the soft tissues being incised. Skin tension lines run vertically along the shin and horizontally at the knee. ${ }^{13}$ Scars that run contrary to the skin tension lines are more likely to require revision due to increased strain and risk of dehiscence or spread. ${ }^{15}$ A visual of surgical site locations and skin tension lines can be seen in Figure 2.

Each of the autografts (BPTB, HS, QT) requires healing of the harvest site and the healing of the ACL graft including bone, cartilage, and soft tissue remodeling. ${ }^{9,11}$ Not all graft sites heal at the same rate; BPTB and QT grafts recover slower than HS and allografts in muscle and tendon strength. ${ }^{14,16}$ Despite a quicker recovery of strength, HS grafts and allografts have been associated with higher retear rates, especially in younger athletes. ${ }^{17}$ While not interdependent, the healing of the fascia, harvest sites, and skin progress concurrently. Gradually increasing weightbearing and progressively increasing the resistance of exercises throughout the range of motion can simultaneously facilitate remodeling of the integumentary and other body systems, including the neuromuscular and musculoskeletal systems. ${ }^{11}$ Weight-bearing progression may have specific implications for the musculoskeletal system, given decreased bone mineral density has been reported across several joints of the lower extremity. ${ }^{18-20}$ Protracted healing and adverse scarring can slow the athletes' progression in multiple facets of rehabilitation (range of motion, locomotion, strength training) due to limited range of motion and pain.

Several methods exist to approximate the skin in order to promote healing. ${ }^{15}$ The most frequent technique after ACLR is closure of the dermis and subcutaneous tissue with absorbable sutures and reinforcement with superficial epidermal sutures, steri-strips or cyanoacrylate adhesive (such as Dermabond; Ethicon Inc, Somerville, NJ). Surgical sutures that are not absorbable are typically removed within two weeks to prevent hash marks (a series of parallel scars connecting suture sites perpendicular to the incision) due to re-epithelialization at the suture puncture sites. Steri- 
strips or similar devices can be used to support healing once sutures are removed.

\section{ASSESSMENT OF THE INTEGUMENTARY SYSTEM}

Assessment of the IS should be performed in a clean, well lit room. The patient's history, including nutrition, smoking history, history of previous scarring or infections, and systemic conditions such as immunosuppression or diabetes should be noted. ${ }^{21}$ Surgical history, including length of surgical procedure, should also be documented, with procedures lasting over two hours at higher risk of infection. ${ }^{22}$ Skin should be observed for incision closure and skin integrity, including areas of discoloration, edema, scarring, nodules, and/or scaly skin. ${ }^{23}$ Any discharge from the incision site should be noted with location, color, and smell. Areas that feel warm should be documented. ${ }^{22}$ Skin mobility should be assessed around the incision and any areas with edema or altered skin integrity should be noted. Signs of infection should be recognized as localized warmth, erythema (redness), localized pain and/or drainage within 30 days of the surgery, most commonly in the first 4-10 days post-operatively. ${ }^{22}$

As the athlete progresses, the incision site should continue to be monitored. Scars can take 1 year or longer to mature. Gentle massage and treatments described below can aid in the remodeling process. ${ }^{24}$ Many athletes report sensation changes around the incision site. ${ }^{17,25,26}$ Sensation after ACLR can be evaluated using pain reported during kneeling, ${ }^{17}$ monofilaments to determine the cutaneous sensation detection thresholds, ${ }^{25}$ and vibratory perception thresholds for vibration sense. ${ }^{26}$ Proper management and treatment of the IS will aid in the athlete's ability to recover after ACLR.

\section{TREATING THE INCISION SITES}

Cutaneous wounds heal in three phases: inflammatory (two to three days), proliferative (two to three weeks) and remodeling (up to 12 months). Creating an optimized environment for wound healing allows these phases to progress appropriately. After surgery, the tensile strength of the skin progresses from $3 \%$ of normal tissue at week one to $20 \%$ at week three and roughly $80 \%$ at week twelve. ${ }^{27}$ Increased age, smoking status and other comorbidities (diabetes, nutritional deficiencies, etc.) that that hinder nutrient and oxygen delivery to wound tissue will prolong healing. ${ }^{28,29}$ If the healing of the incision site is protracted due to bleeding, pain, infection or dehiscence, it could contribute to limitations in achieving range-of-motion goals and adversely affect multiple body systems. ${ }^{29}$

The first 12 days post-operatively are the most important for management of the incision site and prevention of wound dehiscence. ${ }^{28,29}$ Wound care for the incision site is essential for efficient healing and prevention of infection. Surgical sites should be gently cleaned daily with soap and water followed by application of semi-occlusive hydrating emollients, such as petroleum jelly, and a dressing. Emollients can provide a protective barrier as well as hydration to the healing skin. ${ }^{28}$ These interventions promote mobility of the IS and decrease stress on the newly developing

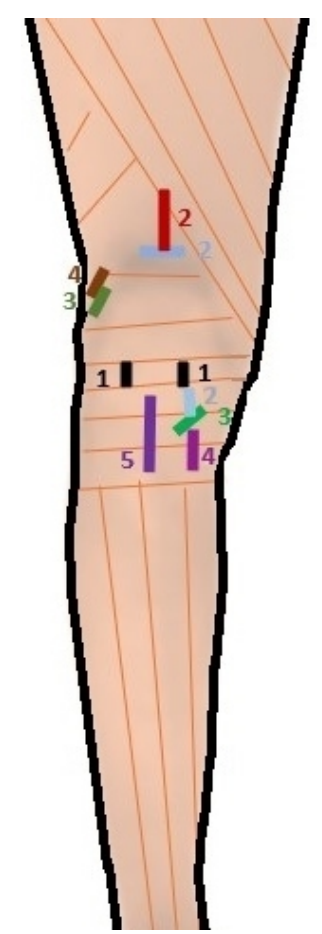

\section{Figure 2. Surgical site locations for the five most common graft types and how they relate to the skin tension lines}

1) fat pad portal sites for all grafts, including the allograft, 2) horizontal and vertical quadriceps tendon grafts above the patella with a potential small secondary site by the pes anserine, $3 \& 4$ ) superior-lateral portal sites for the hamstring grafts with incisions by the pes anserine for the hamstring grafts, 5) large anterior medial incision site for the bone patellar tendon bone graft.

skin. $^{29}$

Patients should avoid submersion into a public water source such as a pool until the incision is completely closed. Hot tubs and other environments that pose a high risk of infection (lakes, rivers) should be avoided until the remodeling phase which starts approximately one month postoperatively. If an infection occurs, systemic antibiotics prescribed by the referring physician are considered the first-line treatment with subsequent operative debridement if the antibiotics are ineffective. ${ }^{30}$ Silicone sheets and gels, as well as scar massage, have shown efficacy in minimizing excess scar tissue formation. ${ }^{31}$ Athletes should also be encouraged to use sunscreen the first year post-operatively to protect the new skin around the incision site.

Providers should be aware that many topical products used during wound care can lead to contact sensitization. Allergy to adhesives (as found in postoperative bandaging), antimicrobial products, and other agents may produce an inflammatory rash that can be confused with infection. It is important to recognize that a geometric distribution of an erythematous, scaly rash (typically very pruritic) with a lack of other infectious signs and symptoms are suggestive of an allergic reaction and can be treated with topical steroids and removal of the offending agent.

Range of motion goals are critical to achieve during this time as well. However, because wound strength is lowest in the early period after surgery, a conservative mobility routine is advised the first weeks. Keeping the incision clean 
and hydrated while maintaining skin mobility and promoting neural regeneration of cutaneous receptors will provide the best results of managing the rehabilitation of the IS following an ACLR. Addressing underlying IS impairments contributes to optimizing the recovery of other physiologic systems after ACLR, which in turn impacts the patent's ability to participate in movement-related interventions.

\section{TREATING SENSORY LOSS}

Due to the knee's use as a kneeling structure and the somatosensory changes throughout the limb after ACLR, superficial nerves of the knee, foot, and ankle, should be evaluated pre- and post-operatively.

Locations around and below the knee may feel numb due to sensory nerve severance during the surgical procedure. These areas can remain numb for weeks, months, or years ${ }^{25}$ after ACLR. The greatest risk for sensation impairments is found along the infrapatellar branch of the saphenous nerve. The graft's incision site is also likely to sever superficial nerves. ${ }^{25,32,33}$ Zones of the leg with decreased sensation should be identified as potential risks of other sensory impairments such as: burns, razor burns (or shaving injuries), poor thermoregulation, hypersensitivity, or altered proprioceptive feedback. ${ }^{34}$ The loss of the native ACL diminishes proprioceptive input and likely affects movement during functional tasks, requiring rehabilitation interventions that target the neuromuscular system.

Sensory integration training should attempt to promote neural regeneration by providing a gradual progression from non-noxious stimuli to more noxious (painful) stimuli, utilizing electrical stimulation and manual therapy interventions to decrease pain and promote healing and mobility of the skin. ${ }^{34}$ Skin care promotes an optimal environment for neural regeneration, starting with electrical stimulation, scar massage, and regular use of emollients. ${ }^{28,34}$ An easy way to progress and promote normal sensation, and potentially help identify neurogenic pain, 35 is for athletes to wear clothes and coverings on the injured leg as soon as possible. Gentle massage can also provide the skin sensory feedback and improve skin mobility. Concerns of chronic pain or hypersensitivity should be communicated with the physician. Conditions such as nerve entrapment, complex regional pain syndrome (CRPS), or chronic pain may require additional surgical corrections or methods of analgesia (nerve block or medication changes) and pain reduction (such as TENS) techniques to reduce the neurogenic pain. ${ }^{34,35}$ Progressive return to less than comfortable activities should be integrated into rehabilitation for sensory normalization as appropriate. Kneeling is an early example of a method to identify abnormal sensations (i.e.: pain) and modulating the intensity (such as moving from foam to hard surfaces and from quadruped to tall kneeling) in order to diminish the painful response. As an athlete progresses, sport-specific activities may be painful, requiring progressive loading or sensory integration to reduce fearavoidance movement patterns secondary to altered afferent feedback related to altered sensation and chronic pain. ${ }^{36}$

\section{ADDRESSING POOR SCARRING}

The two most common scars that concern patients are those that are stretched and atrophic and those that are thickened (i.e. hypertrophic or keloidal scars). Scars that stretch occur when tension on the wound, created by movement of underlying muscles, overcomes the strength of the skin at the line of closure. ${ }^{12}$ These changes are most remarkable in the first eight weeks post-operatively and typically do not worsen after the twelfth week. ${ }^{13}$ Referral to dermatology or plastic surgery for scar revision may be warranted if the patient is displeased with the scar aesthetics.

Hypertrophic scars and keloids are due to excess deposition of collagen at the surgical site. There may be a genetic predisposition for keloid formation thus inquiry into previous wound healing and family history of keloids is important in the preoperative period. ${ }^{37}$ Keloids and hypertrophic scars often are associated with pain or pruritus and are managed with scar massage and silicone sheets. More aggressive therapy includes intralesional steroid injections, intralesional 5-fluorouracil injections, radiation treatments, and cryotherapy, among others. ${ }^{37,38}$ These are typically performed by a dermatologist or plastic surgeon. Frequent conversations should be had with the surgeon with a potential referral warranted if symptoms interfere with the knee's functional movement. As a collaborative practitioner, physical therapists are able to offer a unique perspective on movement-related impairments and interventions to optimize functional capacity and recovery after ACLR. ${ }^{2}$

\section{CONSIDERING HEALTH OF THE PATIENT}

It is important to assess patients' underlying medical conditions and behaviors as pre-existing conditions could prolong or complicate the healing of the incision or scar. ${ }^{28,29,34}$ The skin of younger individuals tends to heal better than older adults after surgery given healthy cardiovascular system and lack of actinic damage (damage from the sun) to the skin. However, these patients are more at risk for hypertrophic or keloidal scars, especially if there is a family or personal history. ${ }^{39}$ Genetic conditions such as hypermobility syndromes (i.e., Ehlers Danlos Syndrome) that alter the normal function of connective tissue elements (i.e. collagen, elastin, etc.) predispose affected patients to the potential for poor wound healing and protracted rehabilitation $^{39}$ and should be considered. Being prepared to address each patient's potential obstacles to rehabilitation will allow providers to tailor programs appropriately and positively impact the recovery of multiple body systems throughout rehabilitation.

\section{CONCLUSION}

The IS contributes to success after ACLR and can provide an early indication of a prolonged rehabilitation process in certain instances, which may ultimately impact other physiologic systems and the patient's ability to participate in movement-related interventions. Creating incisions along skin tension lines, keeping the incision clean and hydrated with appropriate dressings, and regular close assessment 
of wound and scar progression will improve the aesthetic of the scar and limit the potential for poor scar mobility. Furthermore, proper care of the IS can decrease the risk of an infection that could delay or severely dismantle the progress of an individual's ACL rehabilitation. Despite being overlooked at times, the IS plays a crucial role in the functioning of other physiologic systems after ACLR.

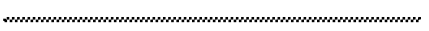

\section{FINANCIAL DISCLOSURES}

The authors have declared no conflict of interest.
FUNDING

None declared.

\section{ACKNOWLEDGEMENTS}

None.

Submitted: October 01, 2021 CST, Accepted: November 01, 2021 CST 


\section{REFERENCES}

1. Mission, Vision, and Strategic Plan. American Physical Therapy Association Website. Accessed June 17, 2021. https://www.apta.org/apta-and-you/leaders hip-and-governance/vision-mission-and-strategic-pl $\underline{\text { an }}$

2. American Physical Therapy Association. An American Physical Therapy Association white paper. Physical therapist practice and the human movement system. Published online 2015:1-4.

3. Saladin L, Voight M. Introduction to the movement system as the foundation for physical therapist practice education and research. Int J Sports Phys Ther. 2017;12(6):858-861. doi:10.16603/ijspt2017085 $\underline{8}$

4. Hedman LD, Quinn L, Gill-Body K, et al. White paper: movement system diagnoses in neurologic physical therapy. J Neurol Phys Ther. 2018;42(2):110-117. doi:10.1097/npt.00000000000002 $\underline{15}$

5. Ludewig PM, Lawrence RL, Braman JP. What's in a name? Using movement system diagnoses versus pathoanatomic diagnoses. J Orthop Sports Phys Ther. 2013;43(5):280-283. doi:10.2519/jospt.2013.0104

6. Quinn L, Riley N, Tyrell CM, et al. A framework for movement analysis of tasks: recommendations from the Academy of Neurologic Physical Therapy's Movement System Task Force. Phys Ther. 2021;101(9). doi:10.1093/ptj/pzab154

7. Gerrett N, Griggs K, Redortier B, Voelcker T, Kondo N, Havenith G. Sweat from gland to skin surface: production, transport, and skin absorption. J Appl Physiol. 2018;125(2):459-469. doi:10.1152/japplphysi ol.00872.2017

8. Jenkins BA, Lumpkin EA. Developing a sense of touch. Development. 2017;144(22):4078-4090. doi:1 $\underline{0.1242 / \text { dev.120402 }}$

9. Beynnon BD, Johnson RJ, Abate JA, Fleming BC, Nichols CE. Treatment of anterior cruciate ligament injuries, part I. Am J Sports Med. 2005;33(10):1579-1602. doi:10.1177/03635465052799 $\underline{13}$

10. van Grinsven S, van Cingel RE, Holla CJ, van Loon CJ. Evidence-based rehabilitation following anterior cruciate ligament reconstruction. Knee Surg Sports Traumatol Arthrosc. 2010;18(8):1128-1144. doi:10.100 7/s00167-009-1027-2
11. Wright RW, Haas AK, Anderson J, et al. Anterior cruciate ligament reconstruction rehabilitation: MOON guidelines. Sports Health. 2015;7(3):239-243. doi:10.1177/1941738113517855

12. Bolognia JL, Jorizzo JL, Schaffer JV. Dermatology. 3rd ed. Elsevier; 2012.

13. Robinson JK. Anatomy for procedural dermatology. In: Robinson JK, Hanke CW, Siegel DM, Fratila A, Bhatia AC, Rohrer TE, eds. Surgery of the Skin. 3rd ed. Elsevier; 2015.

14. Mouarbes D, Menetrey J, Marot V, Courtot L, Berard E, Cavaignac E. Anterior cruciate ligament reconstruction: a systematic review and metaanalysis of outcomes for quadriceps tendon autograft versus bone-patellar tendon-bone and hamstringtendon autografts. Am J Sports Med. 2019;47(14):3531-3540. doi:10.1177/03635465188253 $\underline{40}$

15. Weitzul S, Taylor RS. Suturing technique and other closure materials. In: Robinson JK, Hanke CW, Sengelmann R, Siegel DM, eds. Surgery of the Skin. 1st ed. Mosby; 2005:225-244.

16. Smith AH, Capin JJ, Zarzycki R, Snyder-Mackler L. Athletes with bone-patellar tendon-bone autograft for anterior cruciate ligament reconstruction were slower to meet rehabilitation milestones and returnto-sport criteria than athletes with hamstring tendon autograft or soft tissue allograft: secondary analysis from the ACL-SPORTS trial. J Orthop Sports Phys Ther. 2020;50(5):259-266. doi:10.2519/jospt.2020.9111

17. Widner M, Dunleavy M, Lynch S. Outcomes following ACL reconstruction based on graft type: are all grafts equivalent? Curr Rev Musculoskelet Med. 2019;12(4):460-465. doi:10.1007/s12178-019-0958 $\underline{8-\mathrm{W}}$

18. Nyland J, Fisher B, Brand E, Krupp R, Caborn DN. Osseous deficits after anterior cruciate ligament injury and reconstruction: a systematic literature review with suggestions to improve osseous homeostasis. Arthroscopy. 2010;26(9):1248-1257. do i:10.1016/j.arthro.2010.03.017

19. van Meer BL, Waarsing JH, van Eijsden WA, et al. Bone mineral density changes in the knee following anterior cruciate ligament rupture. Osteoarthritis Cartilage. 2014;22(1):154-161. doi:10.1016/j.joca.201 $\underline{3.11 .005}$ 
20. Reiman MP, Rogers ME, Manske RC. Interlimb differences in lower extremity bone mineral density following anterior cruciate ligament reconstruction. J Orthop Sports Phys Ther. 2006;36(11):837-844. doi:1 $\underline{0.2519 / j o s p t .2006 .2278}$

21. Weinstein MC, Vidimos AT. Patient evaluation, informed consent, preoperative assessment, and care. In: Robinson JK, Hanke CW, Siegel DM, Fratila A, Bhatia AC, Rohrer TE, eds. Surgery of the Skin. 3rd ed. Elsevier; 2015.

22. Marlwalla K. Antibiotics. In: Robinson JK, Hanke CW, Siegel DM, Fratila A, Bhatia AC, Rohrer TE, eds. Surgery of the Skin. 3rd ed. Elsevier; 2015.

23. High WA, Tomasini CF, Argenziano G, Zalaudek I. Basic principles of dermatology. In: Bolognia JL, Schaffer JV, Cerroni L, eds. Dermatology. 4th ed. Elsevier; 2018.

24. Leitenberger JJ, Isenhath SN, Swason NA, Lee KK. Revision of surgical scars. In: Robinson JK, Hanke CW, Siegel DM, Fratila A, Bhatia AC, Rohrer TE, eds. Surgery of the Skin. 3rd ed. Elsevier; 2015.

25. Hoch JM, Perkins WO, Hartman JR, Hoch MC. Somatosensory deficits in post-ACL reconstruction patients: a case-control study. Muscle Nerve. 2017;55(1):5-8. doi:10.1002/mus.25167

26. Cronström A, Ageberg E. Association between sensory function and medio-lateral knee position during functional tasks in patients with anterior cruciate ligament injury. BMC Musculoskelet Disord. 2014;15:430. doi:10.1186/1471-2474-15-430

27. Son D, Harijan A. Overview of surgical scar prevention and management. J Korean Med Sci. 2014;29(6):751-757. doi:10.3346/jkms.2014.29.6.751

28. Jourdan M, Madfes DC, Lima E, Tian Y, Seité S. Skin care management for medical and aesthetic procedures to prevent scarring. Clin Cosmet Investig Dermatol. 2019;12:799-804. doi:10.2147/ccid.S218134

29. Agha R, Ogawa R, Pietramaggiori G, Orgill DP. A review of the role of mechanical forces in cutaneous wound healing. J Surg Res. 2011;171(2):700-708. doi:1 0.1016/i.jss.2011.07.007

30. Judd D, Bottoni C, Kim D, Burke M, Hooker S. Infections following arthroscopic anterior cruciate ligament reconstruction. Arthroscopy. 2006;22(4):375-384. doi:10.1016/j.arthro.2005.12.002
31. Marini L, Odendaal D, Smirnyi S. Importance of scar prevention and treatment-an approach from wound care principles. Dermatol Surg. 2017;43 Suppl 1:S85-s90. doi:10.1097/dss.0000000000001001

32. Grassi A, Perdisa F, Samuelsson K, et al. Association between incision technique for hamstring tendon harvest in anterior cruciate ligament reconstruction and the risk of injury to the infrapatellar branch of the saphenous nerve: a metaanalysis. Knee Surg Sports Traumatol Arthrosc. 2018;26(8):2410-2423. doi:10.1007/s00167-018-485 $\underline{8-\mathrm{X}}$

33. Sharaby MMF, Alfikey A, Alhabsi IS, Al-Ghannami $\mathrm{S}$. No difference in sensory outcome between vertical and oblique incisions for hamstring graft harvest during ACL reconstruction. Knee Surg Sports Traumatol Arthrosc. 2019;27(1):146-152. doi:10.1007/ s00167-018-5057-5

34. Lebonvallet N, Laverdet B, Misery L, Desmoulière A, Girard D. New insights into the roles of myofibroblasts and innervation during skin healing and innovative therapies to improve scar innervation. Exp Dermatol. 2018;27(9):950-958. doi:10.1111/exd.1 $\underline{3681}$

35. Hong AJ, Agarwalla A, Liu JN, et al. Neurological structures and mediators of pain sensation in anterior cruciate ligament reconstruction. Ann Anat. 2019;225:28-32. doi:10.1016/j.aanat.2019.05.010

36. Burland JP, Lepley AS, Cormier M, DiStefano LJ, Arciero R, Lepley LK. Learned helplessness after anterior cruciate ligament reconstruction: an altered neurocognitive state? Sports Med. 2019;49(5):647-657. doi:10.1007/s40279-019-01054-4

37. Ud-Din S, Bayat A. Strategic management of keloid disease in ethnic skin: a structured approach supported by the emerging literature. Br J Dermatol. 2013;169 Suppl 3:71-81. doi:10.1111/bjd.12588

38. Berman B, Maderal A, Raphael B. Keloids and hypertrophic scars: pathophysiology, classification, and treatment. Dermatol Surg. 2017;43 Suppl 1:S3-s18. doi:10.1097/dss.0000000000000819

39. Bolognia JL, Schaffer JV, Cerroni L. Dermatology. 4th ed. Elsevier; 2018. 